\title{
Positive solution to the nonlinear abstract Hammerstein equation and applications to $\phi$-Laplacian BVPs
}

\author{
Abdelhamid Benmezaï and Salima Mechrouk
}

\begin{abstract}
We provide in this paper existence results for positive solution to the abstract Hammerstein equation $N F u=u$ where $N: E \rightarrow E$ is a completely continuous operator, $F: C \rightarrow C$ is a continuous and bounded map and $C$ is a cone in the Banach space $E$. The obtained results are used to prove existence results for positive solution to $\phi$-Laplacian boundary value problem.
\end{abstract}

Mathematics Subject Classification. 47H11, 34B15.

Keywords. Hammerstein equation, Fixed point index theory, BVPs.

\section{Introduction}

Recently and in an interesting paper, García-Huidobro et al. studied in [10] existence of positive solutions to the $\phi$-Laplace boundary value problem

$$
\begin{cases}\left(\phi\left(u^{\prime}\right)\right)+f(t, u), & t \in(0,1) \\ \theta(u(0))=\beta \theta\left(u^{\prime}(0)\right), & \theta(u(1))=-\delta \theta\left(u^{\prime}(1)\right)\end{cases}
$$

where $\beta, \delta$ are nonnegative real numbers, $\phi, \theta$ are odd increasing homeomorphisms from $\mathbb{R}$ onto $\mathbb{R}$ and $f:[0,1] \times[0,+\infty) \rightarrow[0,+\infty)$ is continuous with $f(t, 0)=0, f(t, s)>0$ for all $s>0$ and $t \in[0,1]$. They obtain existence results under one of the following condition

$$
f_{0}(t)=0 \quad \text { and } \quad f_{0}(t)=+\infty
$$

or

$$
f_{0}(t)=+\infty \quad \text { and } \quad f_{0}(t)=0
$$

where for $\nu=0,+\infty, f_{\nu}(t)=\lim _{s \rightarrow \nu} f(t, s) / \phi(s)$. 
Their results and all those in $[3,4]$ and [6-9], are obtained by mean of a fixed point formulation having the form

$$
u=N F u \text {. }
$$

Equation (1) is known as the abstract Hammerstein equation (see chapter 7 in [13]). If nonnegativity is a requirement for a solution to (1) then we are naturely led to use methods of Nonlinear Analysis in ordered Banach spaces. So let $E$ be a Banach space, $K$ a cone in $E, N: E \rightarrow E$ is a completely continuous and increasing operator which is not necessarily linear (the case where $N$ is linear has been studied in [1]), $F: K \rightarrow K$ is a continuous and bounded map. We are interested in this paper by existence and nonexistence results for solutions to Eq. (1) in the cone $K$. Roughly speaking with any cone $P$ such that $N(K) \subset P \subset K$ we will associate two classes of nonlinearities $F$, the first class is constituted by nonlinearities for which equation 1 ) has no solution in $P$ and the second one is constituted by nonlinearities for which equation 1 ) is able to have a solution in $P$ (see Remark 3.6). Since for nonlinearities in the second class we can not claim that Eq. (1) admits a solution in the cone $P$ we provide by Theorems 3.10, 3.11, 3.12 and 3.13 subclasses of nonlinearities $F$ for which Eq. (1) admits a solution in $P$.

As an application, obtained results are used to prove existence results for positive solution to $\phi$-Laplacian boundary value problems.

Throughout this paper, for any subset $A$ of a Banach space, $A^{*}:=$ $A \backslash\{0\}$.

\section{Preliminaries}

We will use extensively in this work cones and the fixed point index theory, so let us recall some facts related to these two tools. Let $X$ be a Banach space, a nonempty closed convex subset $K$ of $X$ is said to be an ordered cone if

- $(t K) \subset K$ for all $t \geq 0$

- $K \cap(-K)=\{0\}$.

It is well known that an ordered cone $K$ induces a partial order in the Banach space $X$. We write for all $x, y \in X: x \leq y$ if $y-x \in K, x<y$ if $y-x \in K, y \neq x$ and $x \not \leq y$ if $y-x \notin K$. Notations $\geq,>$ and $\geq$ denote respectively the inverse situations.

A cone $K$ is said to be normal with a constant $n>0$ if for all $u, v$ in $K, u \leq v$ implies $\|u\| \leq n\|v\|$.

A function $f: \Omega \subset X \rightarrow X$ is said to be bounded if it maps bounded sets into bounded sets and it is said to be completely continuous if it is continuous and maps bounded sets into relatively compact sets.

Now let $K$ be a cone of $E$ and $N: E \rightarrow E$ a continuous map.

Definition 2.1. $N$ is said to be

- positive if $N(K) \subset K$,

- strongly positive if $K$ has a nonempty interior $($ int $K \neq \emptyset)$ and $N\left(K^{*}\right) \subset$ intK, 
- positively 1-homogeneous if for all $u \in E$ and $t \geq 0, N(t u)=t N(u)$,

- increasing if for all $u, v \in E, u \leq v$ implies $N u \leq N v$ and

- strictly increasing if for all $u, v \in E, u<v$ implies $N u<N v$.

Definition 2.2. $N$ is said to be upper bounded on $K$ if there exists a positive constant $M$ such that for all $u \in K,\|N u\| \leq M\|u\|$. In a such situation we denote

$$
N_{K}^{+}=\sup \left\{\|N u\| /\|u\|, u \in K^{*}\right\} .
$$

Definition 2.3. $N$ is said to be lower bounded on $K$ if there exists a positive constant $m$ such that for all $u \in K,\|N u\| \geq m\|u\|$. In a such situation we denote

$$
N_{K}^{-}=\inf \left\{\|N u\| /\|u\|, u \in K^{*}\right\} .
$$

The following result gives a nonlinear version of Krein Rutman theorem,

Theorem 2.4. ([5,12]) Let $N$ be an increasing positively 1-homogeneous completely continuous mapping for which there exists $u \in K^{*}$ and $m>0$ such that $N u \geq m u$, then $T$ has a positive eigen-pair $\left(\lambda_{0}, u_{0}\right)$.

Furthermore if $N$ is strongly positive and strictly increasing then

- if $\lambda$ is an eigenvalue with nonnegative eigenvector, then $\lambda=\lambda_{0}$.

- $\lambda_{0}$ is geometrically simple.

- if $\lambda$ is a real eigenvalue of $N$, then $|\lambda| \leq \lambda_{0}$.

Let $B(0, R)$ be the ball of radius $R>0$ centred at the origin and $f: K \rightarrow$ $K$ a completely continuous map. Following lemmas provide computations of the fixed point index:

Lemma 2.5. If $f x \nsupseteq x$ for all $x \in \partial B(0, R) \cap K$ then

$$
i(f, B(0, R) \cap K, K)=1 \text {. }
$$

Lemma 2.6. If $f x \not \leq x$ for all $x \in \partial B(0, R) \cap K$ then

$$
i(f, B(0, R) \cap K, K)=0 .
$$

Lemma 2.7. If $\|f x\|<\|x\|$ for all $x \in \partial B(0, R) \cap K$ then

$$
i(f, B(0, R) \cap K, K)=1 \text {. }
$$

Lemma 2.8. If $\|f x\|>\|x\|$ for all $x \in \partial B(0, R) \cap K$ then

$$
i(f, B(0, R) \cap K, K)=0 .
$$

For more details and proofs we refer the reader to [11].

\section{Main results}

In all this section $E$ is a Banach space, $C$ a cone of $E$ and $N: E \rightarrow E$ an increasing operator. For any subset $P$ of $C$ with $P^{*}$ nonempty we define the subsets

$\Lambda_{P}^{N}=\left\{\lambda \geq 0\right.$ : there exist $u \in P^{*}$ such that $\left.N u \leq \lambda u\right\}$,

$\Theta_{P}^{N}=\left\{\theta \geq 0\right.$ : there exist $u \in P^{*}$ such that $\left.N u \geq \theta u\right\}$. 
Note that

- $0 \in \Lambda_{P}^{-}$and if $\theta \in \Theta_{P}^{N}$ then $[0, \theta] \subset \Theta_{P}^{N}$.

- If $\lambda \in \Lambda_{P}^{N}$ then $\left[\lambda,+\infty\left[\subset \Lambda_{P}^{N}\right.\right.$.

- if $P \subset K \subset C$ then $\Lambda_{P}^{N} \subset \Lambda_{K}^{N} \subset \Lambda_{C}^{N}$ and $\Theta_{P}^{N} \subset \Theta_{K}^{N} \subset \Theta_{C}^{N}$.

The following constants $\lambda_{P}^{N}$ and $\theta_{P}^{N}$ will play an important role in the statement of the obtained existence and nonexistence results. When they exist, they are defined for any subset $P \subset C$ with $P^{*} \neq \varnothing$ by

$$
\lambda_{P}^{N}=\inf \Lambda_{P}^{N}, \theta_{P}^{N}=\sup \Theta_{P}^{N} .
$$

The following Lemmas 3.1 and 3.3 provide suffiscient condition for existence of $\lambda_{P}^{N}$ and $\theta_{P}^{N}$ respectively.

Lemma 3.1. If $C$ is normal with a constant $n$ and $N$ is upper bounded on $C$ then the subset $\Theta_{C}^{N}$ is bounded from above.

Proof. If $\lambda>0$ and $u \in C^{*}$ are such that $\lambda u \leq N u$ then the upper boundeness of $N$ and the normality of $C$ lead to

$$
\lambda\|u\| \leq n\|N u\| \leq n N_{C}^{+}\|u\|
$$

then

$$
\lambda \leq n N_{C}^{+}
$$

Remark 3.2. See that if $C$ is normal and $N$ is upper bounded on $C$ then for all subset $P \subset C$ with $P^{*}$ nonempty the subset $\Theta_{P}^{N}$ is bounded from above. Also, arguing as in the proof of Lemma 3.1 we can show that for any normal cone $P$ with $N(C) \subset P \subset C$ if $N$ is upper bounded on $P$ then $\Theta_{P}^{N}$ is bounded from above.

Lemma 3.3. Suppose that $N$ is completely continuous, $P$ is a cone of $E$ with $N(C) \subset P \subset C$ and $N$ is upper bounded on $P$ then the subset $\Lambda_{P}^{N}$ is nonempty.

Proof. Let $\lambda>N_{P}^{+}$and $e \in P^{*}$ and consider the equation

$$
u=N_{\lambda}(u, t)
$$

where for all $u \in C$ and $t \in[0,1]$

$$
N_{\lambda}(u, t)=\frac{t}{\lambda} N u+e .
$$

Clearly $N_{\lambda}(P \times[0,1]) \subset P$ and Eq. (2) has no solution in $P \cap \partial B(0, R)$ with $R>\max \left(\frac{N_{P}^{+}}{\lambda-N_{P}^{+}}\|e\|,\|e\|\right)$. Thus by homotopy and normality properties of fixed point index

$$
i\left(N_{\lambda}(\cdot, 1), B(0, R) \cap P, P\right)=i\left(N_{\lambda}(\cdot, 0), B(0, R) \cap P, P\right)=1
$$

and Eq. (2) admits a solution $u \in P^{*} \cap B(0, R)$. We conclude that $\lambda \in \Lambda_{P}^{N}$.

Remark 3.4. If $P$ is a normal cone with a constant $n_{P}$ such that $N(C) \subset P \subset C$ and $N$ is lower bounded on $P$ then $\Lambda_{P}^{N}$ is bounded from below by $n_{p} N_{p}^{-}$. Indeed if $\lambda>0$ and $u \in P^{*}$ are such that $\lambda u \geq N u$ then $\lambda\|u\| \geq n_{P}\|N u\| \geq n N_{P}^{-}\|u\|$. 
Now let us consider the nonlinear abstract Hammerstein equation

$$
N F u=u
$$

where $F: C \rightarrow C$ is a continuous function.

Before presenting existence results for Eq. (3) we need to draw your intention to the following fact. If $N$ admits an eigenvalue $\lambda \geq 0$ with an eigenvector $u \in C^{*}$ then $\lambda_{C}^{N} \leq \theta_{C}^{N}$ and $\lambda \in\left[\lambda_{C}^{N}, \theta_{C}^{N}\right]$. In the following we will prove that for any cone $P$ with $N(C) \subset P \subset C$ if $N$ is completely continuous then having or not a positive eigenvalue we have all time $\lambda_{C}^{N} \leq \theta_{C}^{N}$. To prove this we need following lemmas.

Lemma 3.5. Let $P$ be a cone of $E$ with $N(C) \subset P \subset C$. If one of the following situations

$$
\begin{aligned}
& F u \leq \alpha u \text { for all } u \in P^{*} \text { with } \alpha \theta_{C}^{N}<1 \\
& F u \geq \beta u \text { for all } u \in P^{*} \text { with } \beta \lambda_{P}^{N}>1
\end{aligned}
$$

holds true then Eq. (3) has no solution in $P^{*}$.

Proof. We present the proof in the case where (4) holds the other case is checked similarly. Assume that there is some $u \in P^{*}$ such that $N F u=u$ then from inequality $F u \leq \alpha u$ yields $N v \geq \frac{1}{\alpha} v$ where $v=\alpha u \in P^{*}$ and $\frac{1}{\alpha} \leq \theta_{P}^{N}$ which contradicts $\alpha \theta_{P}^{N}<1$. The Lemma is proved.

Remark 3.6. Note that Lemma 3.5 claims that Eq. (3) with a nonlinearity $F$ lying below the linearity $\left(\theta_{P}^{N}\right)^{-1} u$ or above the linearity $\left(\lambda_{P}^{N}\right)^{-1} u$, admits no solution in $P^{*}$. In another fashion Eq. (3) is able to have a solution in the cone $P$ if the nonlinearity $F$ crosses at least once the linearity $\left(\theta_{P}^{N}\right)^{-1} u$ or the linearity $\left(\lambda_{P}^{N}\right)^{-1} u$.

Lemma 3.7. Let $P$ be a cone of $E$ with $N(C) \subset P \subset C$ and assume that $N$ is completely continuous, $F$ is bounded. If there exist positive real numbers $\alpha, \beta, R_{1}, R_{2}$ with $\alpha \theta_{P}^{N}<1<\beta \lambda_{P}^{N}$ and $R_{1}<R_{2}$ such that one of the following situations

$$
\begin{array}{r}
\left\{\begin{array}{l}
F u \leq \alpha u \text { for all } u \in K \cap \partial B\left(0, R_{1}\right) \\
F u \geq \beta u \text { for all } u \in K \cap \partial B\left(0, R_{2}\right)
\end{array}\right. \\
\left\{\begin{array}{l}
F u \geq \beta u \text { for all } u \in K \cap \partial B\left(0, R_{1}\right) \\
F u \leq \alpha u \text { for all } u \in K \cap \partial B\left(0, R_{2}\right)
\end{array}\right.
\end{array}
$$

holds true, then Eq. (3) admits a positive solution $u$ with $R_{1}<\|u\|<R_{2}$.

Proof. We present the proof in the case where (6) holds, the other case is checked similarly. Assume that there exists $u \in P \cap \partial B\left(0, R_{1}\right)$ such that $N F u \geq$ $u$. Inequality $F u \leq \alpha u$ leads to $N(\alpha u) \geq u$ or $N(v) \geq \frac{1}{\alpha} v$ where $v=$ $\alpha u \in P^{*}$ that is $\frac{1}{\alpha} \leq \theta_{P}^{N}$ which contradicts $\alpha \theta_{P}^{N}<1$. So by Lemma 2.5, $i\left(N F, P \cap B\left(0, R_{1}\right), P\right)=1$. 
If for some $u \in P \cap \partial B\left(0, R_{2}\right), N F u \leq u$. We get from inequality $F u \geq \beta u$ that $N(\beta u) \leq u$ or $N(v) \geq \frac{1}{\beta} v$ where $v=\beta u \in P^{*}$ that is $\frac{1}{\beta} \geq \lambda_{P}^{N}$ which contradicts $\beta \lambda_{P}^{N}>1$. So by Lemma 2.6, $i\left(N F, P \cap B\left(0, R_{2}\right), P\right)=0$.

At the end by excision and solution properties of the fixed point index we obtain

$$
\begin{aligned}
& i\left(N F, P \cap\left(B\left(0, R_{2}\right) \backslash \bar{B}\left(0, R_{1}\right)\right), P\right) \\
& \quad=i\left(N F, P \cap B\left(0, R_{2}\right), P\right)-i\left(N F, P \cap B\left(0, R_{1}\right), P\right)=-1
\end{aligned}
$$

and Eq. (3) admits a positive solution $u$ with $R_{1}<\|u\|<R_{2}$.

Remark 3.8. Arguing as in the proof of Lemma 3.7 one can see that if $N$ is completely continuous and $P$ is a cone of $E$ with $N(C) \subset P \subset C$ then for all $R>0$

$$
i(N(\alpha \cdot), P \cap B(0, R), P)= \begin{cases}1 & \text { if } \alpha \theta_{P}^{N}<1 \\ 0 & \text { if } \alpha \lambda_{P}^{N}>1 .\end{cases}
$$

Theorem 3.9. Let $P$ be a cone with $N(C) \subset P \subset C$ and assume that $N$ is completely continuous then $\lambda_{P}^{N} \leq \theta_{P}^{N}$.

Proof. The case $\lambda_{P}^{N}=0$ is obvious, so assume that $\lambda_{P}^{N}>\theta_{P}^{N} \geq 0$ and consider the function $F: C \rightarrow C$ defined by

$$
F u=\frac{\eta u+\mu\|u\| u}{1+\|u\|}
$$

with $0<\eta<\mu$ and $\eta \lambda_{P}^{N}>1>\mu \theta_{P}^{N}$.

Thus we have

$$
\eta u<F u<\mu u \quad \text { for all } u \in P^{*}
$$

In one hand by Lemma 3.5 Eq. (3) admits no positive solution, in the other, for any $0<R_{1}<R_{2}$ we have

$$
F u \leq \mu u \quad \text { for all } u \in P \cap \partial B\left(0, R_{1}\right) \text { with } \beta \theta_{P}^{N}<1
$$

and

$$
F u \geq \eta u \quad \text { for all } u \in P \cap \partial B\left(0, R_{2}\right) \text { with } \eta \lambda_{P}^{N}>1
$$

and by Lemma $3.7 \mathrm{Eq}$. (3)admits a positive solution. This is impossible so $\lambda_{P}^{N} \leq \theta_{P}^{N}$

The first existence result concerns the case where $N$ is positively 1-homogeneous operator.

Theorem 3.10. Let $P$ be a cone of $E$ with $N(C) \subset P \subset C$ and assume that $N$ is an increasing positively 1-homogeneous completely continuous operator, $C$ is normal and there exist three nonnegative real numbers $\alpha, \beta, \gamma$ and continuous functions $G_{i}: C \rightarrow C i=1,2,3$ such that

$$
\alpha \theta_{P}^{N}<1<\beta \lambda_{P}^{N}
$$


and for all $u \in P^{*}$

$$
\begin{aligned}
F u & \leq \alpha u+G_{1}(u) \\
\beta u-G_{2}(u) & \leq F(u) \leq \gamma u+G_{3}(u) .
\end{aligned}
$$

Then if one of the following situations

$$
\begin{gathered}
G_{1}(u)=\circ(\|u\|) \quad \text { at } 0 \text { and } \\
G_{i}(u)=\circ(\|u\|) \quad \text { at } \infty \text { for } i=2,3 \\
G_{1}(u)=\circ(\|u\|) \text { at } \infty \text { and } \\
G_{i}(u)=\circ(\|u\|) \text { at } 0 \text { for } i=2,3
\end{gathered}
$$

holds true, Eq. (3) admits a positive solution.

Proof. We present the prof in the case where (8) holds, the other case is checked similarly. We have to prove existence of $0<r<R$ such that

$$
\begin{aligned}
i(N F, B(0, r) \cap P, P) & =1 \\
i(N F, B(0, R) \cap P, P) & =0,
\end{aligned}
$$

in such situation, excision and solution properties of fixed point index imply that

$$
\begin{aligned}
& i(N F,(B(0, R) \backslash \bar{B}(0, r)) \cap P, P) \\
& \quad=i(N F, B(0, R) \cap P, P)-i(N F, B(0, r) \cap P, P)=-1
\end{aligned}
$$

and Eq. (3) admits a positive solution $u$ with $r<\|u\|<R$.

Now consider the function $H_{1}:[0,1] \times C \rightarrow C$ defined by $H_{1}(t, u)=$ $t N F u+(1-t) \beta N u$ and let us prove existence of $R>0$ large enough such that for all $t \in[0,1]$ equation $H_{1}(t, u)=u$ has no solution in $\partial B(0, R) \cap P$. By the contrary suppose that for all integer $n \geq 1$ there exist $t_{n} \in[0,1]$ and $u_{n} \in \partial B(0, n) \cap P$ such that

$$
u_{n}=t_{n} N F u_{n}+\left(1-t_{n}\right) \beta N u_{n} .
$$

Note that $v_{n}=\frac{u_{n}}{\left\|u_{n}\right\|} \in \partial B(0,1) \cap P$ and satisfies

$$
v_{n}=t_{n} N\left(\frac{F u_{n}}{\left\|u_{n}\right\|}\right)+\left(1-t_{n}\right) \beta N v_{n} .
$$

Thus the normality of the cone $C$ combined with

$$
\beta v_{n}-\frac{G_{2} u_{n}}{\left\|u_{n}\right\|} \leq \frac{F u_{n}}{\left\|u_{n}\right\|} \leq \gamma v_{n}+\frac{G_{3} u_{n}}{\left\|u_{n}\right\|}
$$

and $G_{i}\left(u_{n}\right)=\circ\left(\left\|u_{n}\right\|\right)$ at $\infty$ for $i=2,3$ yields $\frac{F u_{n}}{\left\|u_{n}\right\|}$ is bounded. Then from the compacity of $N$ we deduce existence of a subsequence denoted also $\left(v_{n}\right)$ which converges to $v \in \partial B(0,1) \cap P$ satisfying $v \geq \beta N v$. That is $\frac{1}{\beta} \geq \lambda_{P}^{N}$ which contradicts $\beta \lambda_{P}^{N}>1$. 
For a such $R>0$ we deduce from homotopy property of fixed point index and Lemma 2.6 that

$$
\begin{aligned}
& i(N F, B(0, R) \cap P, P)=i\left(H_{1}(1, \cdot), B(0, R) \cap P, P\right) \\
& \quad=i\left(H_{1}(0, \cdot), B(0, R) \cap P, P\right)=i(\beta N, B(0, R) \cap P, P)=0 .
\end{aligned}
$$

In similar way, consider the function $H_{2}:[0,1] \times C \rightarrow C$ defined by $H_{2}(t, u)=t N F u+(1-t) \alpha N u$ and let us prove existence of $r>0$ small enough such that for all $t \in[0,1]$ equation $H_{2}(t, u)=u$ has no solution in $\partial B(0, r) \cap P$. By the contrary suppose that for all integer $n \geq 1$ there exist $t_{n} \in[0,1]$ and $u_{n} \in \partial B\left(0, \frac{1}{n}\right) \cap P$ such that

$$
u_{n}=t_{n} N F u_{n}+\left(1-t_{n}\right) \beta N u_{n} .
$$

Note that $v_{n}=\frac{u_{n}}{\left\|u_{n}\right\|} \in \partial B(0,1) \cap P$ and satisfies

$$
v_{n}=t_{n} N\left(\frac{F u_{n}}{\left\|u_{n}\right\|}\right)+\left(1-t_{n}\right) \beta N v_{n} .
$$

Thus the normality of the cone $C$ combined with

$$
\frac{F u_{n}}{\left\|u_{n}\right\|} \leq \alpha v_{n}+\frac{G_{1} u_{n}}{\left\|u_{n}\right\|}
$$

and $G_{1}\left(u_{n}\right)=\circ\left(\left\|u_{n}\right\|\right)$ at 0 yields $\frac{F u_{n}}{\left\|u_{n}\right\|}$ is bounded. Then from the compacity of $N$ we deduce existence of a subsequence denoted also $\left(v_{n}\right)$ which converges to $v \in \partial B(0,1) \cap P$ satisfying $v \leq \alpha N v$. Namely $\frac{1}{\alpha} \leq \theta_{P}^{N}$ which contradicts $\alpha \theta_{P}^{N}<1$.

For a such $r>0$ we deduce from homotopy property of fixed point index and Lemma 2.5 that

$$
\begin{aligned}
& i(N F, B(0, r) \cap P, P)=i\left(H_{2}(1, \cdot), B(0, r) \cap P, P\right) \\
& \quad=i\left(H_{2}(0, \cdot), B(0, r) \cap P, P\right)=i(\alpha N, B(0, r) \cap P, P)=1 .
\end{aligned}
$$

This completes the proof.

Now we will present existence results when $N$ is not necessarily positively 1-homogeneous. Assume that $N$ is completely continuous, $F$ is bounded and $P$ is a cone with $N(C) \subset P \subset C . A: E \rightarrow E$ is a positively 1-homogeneous completely continuous increasing operator such that $A(C) \subset P$ which is uniformly continuous on the unit ball of $E$.

Theorem 3.11. Assume that $C$ is normal with a constant $n, N$ is upper bounded on $C$ and there exist three positive real numbers $r, \alpha, \beta$ with $\beta \lambda_{P}^{N}>1>\alpha n N_{C}^{+}$ and a continuous function $G: C \rightarrow C$ with $G(u)=\circ(\|u\|)$ at $\infty$ such that

$$
\begin{aligned}
& F u \geq \beta u \text { for all } u \in P \cap \partial B(0, r) \\
& F u \leq \alpha u+G(u) \text { for all } u \in P,
\end{aligned}
$$

then Eq. (3) admits at least one positive solution. 
Proof. As in proof of Lemma 3.7 we have $i(N F, B(0, r) \cap P, P)=0$ thus it remains to prove that there exists $R>r$ such that $i(N F, B(0, R) \cap P, P)=1$. In view of Lemma 2.7 and by the contrary assume that there is a sequence $\left(u_{n}\right)$ with $u_{n} \in P \cap \partial B(0, n)$ such that $\left\|u_{n}\right\| \leq\left\|N F u_{n}\right\|$, we get then

$$
\left\|u_{n}\right\| \leq\left\|N F u_{n}\right\| \leq n\left\|N\left(\alpha u_{n}+G\left(u_{n}\right)\right)\right\| \leq n N_{C}^{+}\left\|\alpha u_{n}+G\left(u_{n}\right)\right\| .
$$

In another fashion

$$
n N_{C}^{+}\left\|\alpha \frac{u_{n}}{\left\|u_{n}\right\|}+\frac{G\left(u_{n}\right)}{\left\|u_{n}\right\|}\right\|<1 .
$$

Letting $n \rightarrow \infty$ we get the contradiction $1<n N_{C}^{+} \alpha \leq 1$. Hence there exists $R>r$ such that $i(N F, B(0, R) \cap P, P)=0$ and by excision property of the fixed point index $i(N F,(B(0, R) \backslash B(0, r)) \cap P, P)=1$ and Eq. (3) admits at least one solution in $(B(0, R) \backslash \bar{B}(0, r)) \cap P$.

Theorem 3.12. Assume that $A$ is lower bounded on $P$ and

$$
N u=A(u)+\circ(\|u\|) \text { at } \infty .
$$

If there exist three positive real numbers $r, \alpha, \beta$ and $a(\|u\|)$ at $\infty$ such that the following condition

$$
\begin{aligned}
& F u \geq \beta u \quad \text { for all } u \in C \cap \partial B(0, r), \\
& F u \leq \alpha u+G u \text { for all } u \in C \text { and } \\
& \beta \lambda_{P}^{N}>1>\alpha \max \left(\theta_{P}^{N}, \theta_{P}^{A}\right)
\end{aligned}
$$

is satisfied then Eq. (3) admits at least one positive solution.

Proof. As in proof of Lemma 3.7 we have $i(N F, B(0, r) \cap P, P)=0$ thus it remains to prove that there exists $R>r$ such that $i(N F, B(0, R) \cap P, P)=1$. Consider the function $H_{3}:[0,1] \times C \rightarrow C$ defined by $H_{3}(t, u)=t N F u+$ $(1-t) N(\beta u)$ and we claim that there is $R>0$ large enough such that for all $t \in[0,1]$ equation $H_{3}(t, u)=u$ has no solution in $\partial B(0, R) \cap P$. If it is not the case then for all integer $n \geq 1$ there exist $t_{n} \in[0,1]$ and $u_{n} \in \partial B(0, n) \cap P$ such that

$$
u_{n}=t_{n} N F u_{n}+\left(1-t_{n}\right) N\left(\alpha u_{n}\right)
$$

This implies

$$
\begin{aligned}
A\left(\frac{u_{n}}{\left\|u_{n}\right\|}\right)= & A\left(t_{n} \frac{N\left(F u_{n}\right)}{\left\|u_{n}\right\|}+\left(1-t_{n}\right) \alpha \frac{N\left(\alpha u_{n}\right)}{\left\|\alpha u_{n}\right\|}\right) \\
\leq & A\left(t_{n} \frac{N\left(\alpha u_{n}+G u_{n}\right)}{\left\|u_{n}\right\|}+\left(1-t_{n}\right) \alpha \frac{N\left(\alpha u_{n}\right)}{\left\|\alpha u_{n}\right\|}\right) \\
= & A\left(t_{n} \frac{\left\|\alpha u_{n}+G u_{n}\right\|}{\left\|u_{n}\right\|} A\left(\frac{\alpha u_{n}+G u_{n}}{\left\|\alpha u_{n}+G u_{n}\right\|}\right)\right. \\
& \left.+\left(1-t_{n}\right) \alpha A\left(\frac{u_{n}}{\left\|u_{n}\right\|}\right)+\circ(1)\right)
\end{aligned}
$$


From the compacity and the uniform continuity of the operator $A$ we deduce existence of a subsequence $\left(\frac{u_{n_{k}}}{\left\|u_{n_{k}}\right\|}\right)$ such that

$$
\lim A\left(\frac{u_{n_{k}}}{\left\|u_{n_{k}}\right\|}\right)=\lim A\left(\frac{\alpha u_{n}+G u_{n}}{\left\|\alpha u_{n}+G u_{n}\right\|}\right)=v \in P \quad \text { and } \quad v \geq A(\alpha v) .
$$

Furthermore and since $\frac{u_{n_{k}}}{\left\|u_{n_{k}}\right\|} \in P$ and $A$ is lower bounded on $P$,

$$
\|v\|=\lim \left\|A\left(\frac{u_{n_{k}}}{\left\|u_{n_{k}}\right\|}\right)\right\| \geq \lim A_{P}^{-}\left\|\frac{u_{n_{k}}}{\left\|u_{n_{k}}\right\|}\right\|=A_{P}^{-}>0
$$

then we have $w=\alpha v \in C^{*}$ satisfies

$$
A w \geq \frac{w}{\alpha}
$$

and $\frac{1}{\alpha} \leq \theta_{P}^{A}$ which contradicts $\alpha \theta_{P}^{A}<1$.

So there exists $R>r$ such that equation $H_{3}(t, u)=u$ has no solution in $[0,1] \times(\partial B(0, R) \cap P)$ by homotopy property of the fixed point index

$$
i(N F, P \cap B(0, R), P)=i(N(\beta \cdot), P \cap B(0, R), P)=1 .
$$

At the end by excision property of the fixed point index $i(N F,(B(0, R) \backslash$ $B(0, r)) \cap P, P)=1$ and Eq. (3) admits at least one solution in $(B(0, R) \backslash$ $\bar{B}(0, r)) \cap P$.

Theorem 3.13. Assume that $C$ is normal, $A$ is lower bounded on $P$ and

$$
N u=A(u)+\circ(\|u\|) \text { at } \infty .
$$

If there exist four positive real numbers $r, \alpha, \beta, \delta$ and a continuous functions $G_{1}, G_{2}: C \rightarrow C$ with $G_{i}(u)=\circ(\|u\|)$ at $\infty$ for $i=1,2$ such that the following condition

$$
\begin{aligned}
& F u \leq \alpha u \text { for all } u \in C \cap \partial B(0, r), \\
& \beta u-G_{1} u \leq F u \leq \delta u+G_{2} u \quad \text { for all } u \in C \text { and } \\
& \beta \min \left(\lambda_{P}^{A}, \lambda_{P}^{N}\right)>1>\alpha \theta_{P}^{N}
\end{aligned}
$$

is satisfied then Eq. (3) admits at least one positive solution.

Proof. As in proof of Lemma 3.7 we have $i(N F, B(0, r) \cap P, P)=1$ thus it remains to prove that there exists $R>r$ such that $i(N F, B(0, R) \cap P, P)=0$. Consider the function $H_{4}:[0,1] \times C \rightarrow C$ defined by $H_{4}(t, u)=t N F u+$ $(1-t) N(\beta u)$ and we claim that there is $R>0$ large enough such that for all $t \in[0,1]$ equation $H_{4}(t, u)=u$ has no solution in $\partial B(0, R) \cap P$. If it is not the case then for all integer $n \geq 1$ there exist $t_{n} \in[0,1]$ and $u_{n} \in \partial B(0, n) \cap P$ such that

$$
u_{n}=t_{n} N F u_{n}+\left(1-t_{n}\right) N\left(\beta u_{n}\right) .
$$


This implies

$$
\begin{aligned}
A\left(\frac{u_{n}}{\left\|u_{n}\right\|}\right) & =A\left(t_{n} \beta \frac{N\left(F u_{n}\right)}{\left\|\beta u_{n}\right\|}+\left(1-t_{n}\right) \beta \frac{N\left(\beta u_{n}\right)}{\left\|\beta u_{n}\right\|}\right) \\
& =A\left(t_{n} \beta A\left(\frac{F u_{n}}{\left\|\beta u_{n}\right\|}\right)+\left(1-t_{n}\right) \beta A\left(\frac{u_{n}}{\left\|u_{n}\right\|}\right)+\circ(1)\right)
\end{aligned}
$$

Since $C$ is normal and

$$
\frac{F u_{n}}{\left\|\beta u_{n}\right\|} \leq \frac{\delta}{\beta} \frac{u_{n}}{\left\|u_{n}\right\|}+\frac{G_{2} u_{n}}{\left\|\beta u_{n}\right\|},
$$

$\frac{F u_{n}}{\left\|\beta u_{n}\right\|}$ is bounded and from the compacity of $A$ we deduce existence of a subsequence $\left(\frac{u_{n_{k}}}{\left\|u_{n_{k}}\right\|}\right)$ such that

$$
\lim A\left(\frac{u_{n_{k}}}{\left\|u_{n_{k}}\right\|}\right)=v, \quad \lim A\left(\frac{F u_{n}}{\left\|\beta u_{n}\right\|}\right)=w .
$$

Furthermore and since $\frac{u_{n_{k}}}{\left\|u_{n_{k}}\right\|} \in P$ and $A$ is lower bounded on $P$,

$$
\|v\|=\lim \left\|A\left(\frac{u_{n_{k}}}{\left\|u_{n_{k}}\right\|}\right)\right\| \geq \lim A_{P}^{-}\left\|\frac{u_{n_{k}}}{\left\|u_{n_{k}}\right\|}\right\|=A_{P}^{-}>0 .
$$

Also, from the uniform continuity of the operator $A$

$$
w=\lim A\left(\frac{F u_{n}}{\left\|\beta u_{n}\right\|}\right) \geq \lim A\left(\frac{u_{n}}{\left\|u_{n}\right\|}-\frac{G_{1} u_{n}}{\left\|\beta u_{n}\right\|}\right) \geq v .
$$

From all the above we deduce that $\varpi=\beta v$ satisfies

$$
A(\varpi) \leq \frac{\varpi}{\beta}
$$

and $\frac{1}{\beta} \geq \lambda_{P}^{A}$ which contradicts $\beta \lambda_{P}^{A}>1$.

So there exists $R>r$ such that equation $H_{4}(t, u)=u$ has no solution in $[0,1] \times(\partial B(0, R) \cap P)$ by homotopy property of the fixed point index

$$
i(N F, P \cap B(0, R), P)=i(N(\beta \cdot), P \cap B(0, R), P)=1 .
$$

At the end by excision property of the fixed point index $i(N F,(B(0, R) \backslash$ $B(0, r)) \cap P, P)=1$ and Eq. (3) admits at least one solution in $(B(0, R) \backslash$ $\bar{B}(0, r)) \cap P$.

Remark 3.14. If $P \subset K \subset C$ are two cones then $\left[\lambda_{P}^{N}, \theta_{P}^{N}\right] \subset\left[\lambda_{K}^{N}, \theta_{K}^{N}\right] \subset$ $\left[\lambda_{C}^{N}, \theta_{C}^{N}\right]$. This means that the interval $\left[\left(\theta_{P}^{N}\right)^{-1},\left(\lambda_{P}^{N}\right)^{-1}\right]$ becomes the smallest if the cone $P$ is the smallest one containing $N(C)$ and in a such case the above existence and nonexistence results are more accurate. Moreover if $N^{-1}(0) \cap C^{*}=\{0\}$ then for all cone $P$ with $N(C) \subset P \subset C, \Lambda_{P}^{N}=\Lambda_{C}^{N}$, $\Theta_{P}^{N}=\Theta_{C}^{N}$ and so $\lambda_{P}^{N}=\lambda_{C}^{N}, \theta_{P}^{N}=\theta_{C}^{N}$. Indeed if $\lambda>0$ and $u \in P^{*}$ are such that $N u \leq \lambda u$ (resp. $N u \geq \lambda u$ ) then $w=\frac{N u}{\lambda}$ satisfies $N w \leq \lambda w$ (resp. $N w \geq \lambda w)$. 
In the end of this section we present a situation where constants $\lambda_{C}^{N}$ and $\lambda_{C}^{N}$ meet the unique positive eigenvalue of $N$.

Theorem 3.15. Let $E$ and $X$ be two real Banach spaces with $X \subset E, C$ a normal cone in $E$ such that $C_{X}=X \cap F$ is a cone in $F$ with int $t_{X}\left(C_{X}\right) \neq \emptyset$ and $N: E \rightarrow E$ an increasing positively 1-homogeneous completely continuous operator such that $N(E) \subset X, N^{-1}(0) \cap C=\{0\}$ and $N_{X}: X \rightarrow X$ is a strongly positive, strictly increasing and completely continuous operator. If $\lambda_{C}^{N}>0$ then $\lambda_{C}^{N}=\theta_{C}^{N}$ and $\lambda_{N}=\lambda_{C}^{N}=\theta_{C}^{N}$ is the unique positive eigenvalue of $N$.

Proof. First, taking in account Remark 3.14 and Theorem 3.9, conditions $N^{-1}(0) \cap C=\{0\}$ and $\lambda_{C}^{N}>0$ imply that $\theta_{C_{X}}^{N}>\lambda_{C_{X}}^{N}>0$ then there exists $m>0$ and $v_{m} \in C_{X}^{*}$ such that $N v_{m} \geq m v_{m}$. Thus, it follows from Theorem 2.4 that $N_{X}$ admits a unique positive eigenvalue $\lambda_{N}$ and clearly

$$
0<\lambda_{C}^{N}=\lambda_{C_{F}}^{N} \leq \lambda_{N} \leq \theta_{C_{F}}^{N}=\theta_{C}^{N} .
$$

Let

$$
\lambda_{\text {int }_{X}\left(C_{X}\right)}^{N}=\inf \left\{\lambda \geq 0, \exists u \in \operatorname{int}_{X}\left(C_{X}\right) \text { with } N u \leq \lambda u\right\}
$$

and let us prove that $\lambda_{C}^{N}=\lambda_{C_{F}}^{N}=\lambda_{i n t_{X}\left(C_{X}\right)}^{N}$. To this aim let us prove that $\Lambda_{C_{X}}^{N} \subset \Lambda_{\text {int }_{X}\left(C_{X}\right)}^{N}$. If $\lambda \in \Lambda_{C_{X}}^{N}$ then there exists $u \in C_{X}^{*}$ such that $N u \leq \lambda u$ and we distinguish two cases:

- $u \in \operatorname{int}_{X}\left(C_{X}\right)$ in this case $\lambda \in \Lambda_{i n t_{X}\left(C_{X}\right)}^{N}$

- $u \in \partial_{X} C_{X}$ in this case $U=N u \in$ int $_{x} C_{X}$ and satisfies $N U \leq \lambda U$. This again implies $\lambda \in \Lambda_{\text {int }_{X}\left(C_{X}\right)}^{N}$.

We claim that $\theta_{C_{F}}^{N} \leq \lambda_{i n t_{X}\left(C_{X}\right)}^{N}$, indeed if $\lambda_{i n t_{X}\left(C_{X}\right)}^{N}<\theta_{C_{X}}^{N}$ and $\lambda \in$ $\left(\lambda_{\text {int }_{X}\left(C_{X}\right)}^{N}, \theta_{C_{X}}^{N}\right)$ then for a such $\lambda$, there exist $u \in \operatorname{int}_{F}\left(C_{F}\right)$ and $v \in C_{X}^{*}$ such that $N u \leq \lambda u$ and $N v \geq \lambda v$. The fact $u \in \operatorname{int}_{X}\left(C_{X}\right)$ implies existence of $t>0$ small enough such that $u>v_{t}=t v$. Let $K=\left\{w \in C, v_{t} \leq w \leq u\right\}$, it is clear that $K$ is a convex bounded closed set in $E$ and $T=\frac{N}{\lambda}$ maps $K$ into $K$. So Schauder fixed point Theorem guarantee existence of a fixed point $w_{\lambda} \in K$ of $T$ and $\lambda$ is an eigenvalue of $N$. This means all $\lambda \in\left(\lambda_{\text {int }_{X}\left(C_{X}\right)}^{N}, \theta_{C_{X}}^{N}\right)$ are positive eigenvalues of $N$ then of $N_{X}\left(N\left(N w_{\lambda}\right)=\lambda N w_{\lambda}\right.$ with $\left.N w_{\lambda} \in C_{F}\right)$ and contradicts unicity of $\lambda_{N}$. At the end we have from all the above

$$
0<\lambda_{C}^{N}=\lambda_{C_{X}}^{N} \leq \lambda_{N} \leq \theta_{C_{X}}^{N}=\theta_{C}^{N} \leq \lambda_{\text {int }_{X}\left(C_{X}\right)}^{N}=\lambda_{C}^{N}=\lambda_{C_{X}}^{N}
$$

which implies $\lambda_{N}=\lambda_{C}^{N}=\theta_{C}^{N}$ is the unique positive eigenvalue of $N$.

\section{Application to $\Phi$-Laplacian BVPs}

In all this section, $E$ is the Banach space of all continuous functions defined on $[0,1]$ equipped with its sup-norm denoted $\|\cdot\|, C$ is the normal cone of nonnegative functions in $E$ and $u \in E$ is said to be positive if $u \in C^{*}, f:[0,1] \times \mathbb{R}^{+} \rightarrow$ 
$\mathbb{R}^{+}$is continuous, $a:[0,1] \rightarrow \mathbb{R}^{+}$is continuous and don't vanishes identically on any subinterval of $[0,1]$ and $\phi$ is an odd increasing homeomorphism of $\mathbb{R}$.

Throughout we assume that

$$
\begin{aligned}
& \exists \alpha, \beta \in \mathbb{R} \text { with } 0<\alpha<\beta \text { such that } \\
& t^{\beta} \phi(x) \leq \phi(t x) \leq t^{\alpha} \phi(x) \text { for all } x \geq 0 \text { and } t \in(0,1) .
\end{aligned}
$$

In what follows $\psi$ is the inverse function of $\phi$ and we have from(12)

$$
t^{\frac{1}{\alpha}} \psi(x) \leq \psi(t x) \leq t^{\frac{1}{\beta}} \psi(x) \text { for all } x \geq 0 \text { and } t \in(0,1) .
$$

Let $\psi^{+}, \psi^{-}$be the function defined on $\mathbb{R}^{+}$by

$$
\psi^{+}(x)=\left\{\begin{array}{ll}
x^{\frac{1}{\beta}} & \text { if } x \leq 1 \\
x^{\frac{1}{\alpha}} & \text { if } x \geq 1
\end{array} \quad \psi^{-}(x)= \begin{cases}x^{\frac{1}{\alpha}} & \text { if } x \leq 1 \\
x^{\frac{1}{\beta}} & \text { if } x \geq 1 .\end{cases}\right.
$$

It follows from (13) that for all $t \geq 0$ and $x \geq 0$

$$
\psi^{-}(t) \psi(x) \leq \psi(t x) \leq \psi^{+}(t) \psi(x) .
$$

Remark 4.1. Note that if $\phi$ satisfies (12) then $\phi$ satisfies the conditions of Theorems 1.1 and 1.2 in [10].

Let $F: C \rightarrow C$ be the operator defined for $u \in C$ by

$$
F u(x)=\psi(f(x, u(x))) \text { for all } x \in[0,1] .
$$

It is easy to see that $F$ is continuous and bounded (maps bounded sets into bounded sets). We set

$$
\begin{aligned}
f^{0} & =\limsup _{u \rightarrow 0}\left(\max _{t \in[0,1]} \frac{\psi(f(t, u))}{u}\right) f^{\infty}=\limsup _{u \rightarrow+\infty}\left(\max _{t \in[0,1]} \frac{\psi(f(t, u))}{u}\right) \\
f_{0} & =\liminf _{u \rightarrow 0}\left(\min _{t \in[0,1]} \frac{\psi(f(t, u))}{u}\right) f_{\infty}=\liminf _{u \rightarrow+\infty}\left(\min _{t \in[0,1]} \frac{\psi(f(t, u))}{u}\right)
\end{aligned}
$$

and

$$
\begin{aligned}
l^{0} & =\limsup _{u \rightarrow 0}\left(\max _{t \in[0,1]} \frac{f(t, u)}{\phi(u)}\right) l^{\infty}=\limsup _{u \rightarrow+\infty}\left(\max _{t \in[0,1]} \frac{f(t, u)}{\phi(u)}\right) \\
l_{0} & =\liminf _{u \rightarrow 0}\left(\min _{t \in[0,1]} \frac{f(t, u)}{\phi(u)}\right) l_{\infty}=\liminf _{u \rightarrow+\infty}\left(\min _{t \in[0,1]} \frac{f(t, u)}{\phi(u)}\right) .
\end{aligned}
$$

Remark 4.2. It is easy to see that if $\phi$ is homogeneous then $l^{0}=f^{0}, l_{0}=f_{0}$ and so.

\section{1. $\phi$-Laplacian BVP with Dirichlet boundary conditions}

Consider the boundary value problem

$$
\left\{\begin{array}{l}
-\left(\phi\left(u^{\prime}\right)\right)(t)=a(t) f(t, u(t)) \quad t \in(0,1) \\
u(0)=u(1)=0 .
\end{array}\right.
$$

Existence results for positive solutions to $\phi$-Laplacian BVP with Dirichlet boundary conditions have been obtained in [3,4] by Krasnosel'skii or Leggett William fixed Theorems and by the degree theory in [10]. We will use here existence results of section 3 to obtain existence results for positive solutions to Problem (15). 
Let

$$
X=\left\{u \in C^{1}([0,1]), u(0)=u(1)=0\right\}
$$

equipped with the $C^{1}$-norm denoted $\|\cdot\|_{1}\left(\right.$ for $\left.u \in X,\|u\|_{1}=\max \left(\|u\|,\left\|u^{\prime}\right\|\right)\right)$. From Ascoli Arzela Theorem the embedding $i: X \rightarrow E$ is compact. Let $C$ be the normal cone of nonnegative functions in $E$ and $u \in E$ is said to be positive if $u \in C^{*}$. It is easy to see that

$$
P=\{u \in C, u(x) \geq \rho(x)\|u\| \forall x \in[0,1]\}
$$

is a cone in $E$ where for $x \in[0,1], \rho(x)=\min \{x, 1-x\}$ and $C_{X}=C \cap X=$ $i^{-1}(C)$ is a cone in $X$.

Let $N: E \rightarrow E$ be the operator defined for $h \in E$ by

$$
N h(x)=\int_{0}^{x} \psi\left(c(h)-\int_{0}^{t} a(s) \phi(h(s)) d s\right) d t \text { for all } x \in[0,1]
$$

where $c(h)$ is the unique solution of

$$
\int_{0}^{1} \psi\left(c-\int_{0}^{t} a(s) \phi(h(s)) d s\right) d t=0 .
$$

Let $N_{X}: X \rightarrow X$ be the restriction of $N$ to $X, \Phi_{E}: E \rightarrow L^{1}(0,1)$ defined for $h \in E$ by $\Phi_{E}(h)(x)=\phi(h(x)), \Phi_{X}: X \rightarrow L^{1}(0,1)$ the restriction of $\Phi_{E}$ to $X$ and $N_{1}: L^{1}(0,1) \rightarrow X$ the operator defined for $h \in L^{1}(0,1)$ by

$$
N_{1} h(x)=\int_{0}^{x} \psi\left(c_{1}(h)-\int_{0}^{t} a(s) h(s) d s\right) d t \text { for all } x \in[0,1]
$$

where $c_{1}(h)$ is the unique solution of

$$
\int_{0}^{1} \psi\left(c-\int_{0}^{t} a(s) h(s) d s\right) d t=0 .
$$

It is easy to see that $N_{X}=N_{1} \circ \Phi_{X}$ and $N=i \circ N_{1} \circ \Phi_{E}$ and each of $\Phi_{X}$ and $\Phi_{E}$ are continuous bounded maps. So, we deduce from Lemma 2.2 in [6] (see also Section 3 in [10]) that $N$ and $N_{X}$ are a completely continuous operators and $u$ is a positive solution to (15) if and only if $u$ is a fixed point of the completely continuous operator $N F$.

Lemma 4.3. The operator $N$ has the following properties:

1. $N^{-1}(0)=\{0\}$,

2. $N(C) \subset P$,

3. $N$ is strictly increasing,

4. $N$ is upper bounded on $C$ and lower bounded on $P$.

Proof. 1. Obvious.

2. Note that for any $h \in C, v=N h$ is concave and if $t_{h} \in[0,1]$ is such that $\|v\|=v\left(t_{h}\right)$ then we have $\cdot$ if $t_{0} \in(0,1)$,

$$
\begin{aligned}
v(x) & =v\left(\left(\frac{x}{t_{0}}\right) t_{0}+\left(1-\left(\frac{x}{t_{0}}\right)\right) 0\right) \geq\left(\frac{x}{t_{0}}\right) v\left(t_{0}\right)+\left(1-\left(\frac{x}{t_{0}}\right)\right) v(0) \\
& \geq \rho(x)\|v\|
\end{aligned}
$$


for all $x \in\left[0, t_{0}\right]$ and

$$
\begin{aligned}
v(x) & =v\left(\left(\frac{1-x}{1-t_{0}}\right) t_{0}+\left(\frac{x-t_{0}}{1-t_{0}}\right)\right) \geq\left(\frac{1-x}{1-t_{0}}\right) v\left(t_{0}\right)+\left(\frac{x-t_{0}}{1-t_{0}}\right) v(1) \\
& \geq \rho(x)\|v\|
\end{aligned}
$$

for all $x \in\left[t_{0}, 1\right]$,

$$
\begin{aligned}
& \text { if } t_{0}=0 \text { or } 1 \\
& v(x)=v(x+(1-x) 0) \geq x v(1)+(1-x) v(0) \geq \rho(x)\|v\|
\end{aligned}
$$

for all $x \in[0,1]$. The above shows that $N(C) \subset P$.

3. Let $u, v \in E$ with $u<v$ and set $U=N u, V=N v$ and $W=V-U$. Assume that for some $t_{0} \in(0,1), W\left(t_{0}\right)<0$ and let $t_{*} \in(0,1)$ be such that

$$
W\left(t_{*}\right)=\min _{t \in[0,1]} W(t) \quad \text { and } \quad W^{\prime}\left(t_{*}\right)=0 .
$$

In one hand, it follows from the boundary conditions that there exist $t_{1}, t_{2} \in(0,1)$ such that

$$
t_{1}<t_{*}<t_{2} \quad \text { and } \quad W^{\prime}\left(t_{1}\right)<W^{\prime}\left(t_{*}\right)=0<W^{\prime}\left(t_{2}\right) .
$$

In the other hand, we have

$$
-\left(\phi\left(V^{\prime}\right)-\phi\left(U^{\prime}\right)\right)^{\prime}=a(\phi(v)-\phi(u)) \geq 0 \quad \text { on }[0,1]
$$

which implies $\phi\left(V^{\prime}\right)-\phi\left(U^{\prime}\right)$ is nonincreasing on $[0,1]$. In another fashion

$$
\left(\phi\left(V^{\prime}\right)-\phi\left(U^{\prime}\right)\right) W^{\prime}(t) \leq 0 \text { for all } t \in[0,1]
$$

from which follows

$$
\left(\phi\left(V^{\prime}\left(t_{2}\right)\right)-\phi\left(U^{\prime}\left(t_{2}\right)\right)\right) \leq 0 \leq\left(\phi\left(V^{\prime}\left(t_{1}\right)\right)-\phi\left(U^{\prime}\left(t_{1}\right)\right)\right),
$$

then

$$
\left.W\left(t_{2}\right)=V^{\prime}\left(t_{2}\right)\right)-U^{\prime}\left(t_{2}\right) \leq 0 \leq V^{\prime}\left(t_{1}\right)-U^{\prime}\left(t_{1}\right)=W\left(t_{1}\right)
$$

which contradicts (16).

Now if $U=V$ in $[0,1]$ then we get

$$
a(s)(\phi(u(s))-\phi(v(s)))=0 \quad \text { for all } s \in[0,1]
$$

which implies since $a$ don't vanishes on any subinterval of $[0,1]$, that $u(s)=v(s)$ for all $s \in[0,1]$ and contradicts $u<v$.

4. Let $u \in P$ and $t_{u} \in[0,1]$ be such that $N u\left(t_{u}\right)=\|N u\|$, we have

$$
\begin{aligned}
\|N u\|=N u\left(t_{u}\right) & =\int_{0}^{t_{u}} \psi\left(\int_{t}^{t_{u}} a(s) \phi(u(s)) d s\right) d t \\
& =\int_{t_{u}}^{1} \psi\left(\int_{t_{u}}^{t} a(s) \phi(u(s)) d s\right) d t
\end{aligned}
$$

then

$$
\|N u\| \leq \int_{0}^{t_{u}} \psi\left(\left(t_{u}-t\right)\|a\| \phi(\|u\|)\right) d t \leq \frac{\beta}{\beta+1} \psi^{+}(\|a\|)\left(t_{u}\right)^{\frac{\beta+1}{\beta}}\|u\|
$$


and

$\|N u\| \leq \int_{t_{u}}^{1} \psi\left(\left(t-t_{u}\right)\|a\| \phi(\|u\|)\right) d t \leq \frac{\beta}{\beta+1} \psi^{+}(\|a\|)\left(1-t_{u}\right)^{\frac{\beta+1}{\beta}}\|u\|$.

We conclude from (17) and (18) that for all $u \in C$

$$
\|N u\| \leq \frac{\beta}{\beta+1} \min \left(\left(t_{u}\right)^{\frac{\beta+1}{\beta}},\left(1-t_{u}\right)^{\frac{\beta+1}{\beta}}\right) \psi^{+}(\|a\|)\|u\| \leq D_{\phi}^{+}\|u\|
$$

where

$$
D_{\phi}^{+}=\frac{\beta}{\beta+1}\left(\frac{1}{2}\right)^{\frac{\beta+1}{\beta}} \psi^{+}(\|a\|) .
$$

Now if $t_{u} \leq \frac{1}{2}$ we get

$$
\begin{aligned}
\|N u\| & =N u\left(t_{u}\right)=\int_{t_{u}}^{1} \psi\left(\int_{t_{u}}^{t} a(s) \phi(u(s)) d s\right) d t \\
& \geq \int_{\frac{1}{2}}^{1} \psi\left(\int_{\frac{1}{2}}^{t} a(s) \phi(u(s)) d s\right) d t \\
& \geq\|u\| \psi^{-}(\|a\|) \int_{\frac{1}{2}}^{1}\left(\int_{\frac{1}{2}}^{t}(1-s)^{\beta} \frac{a(s)}{\|a\|} d s\right)^{\frac{1}{\alpha}} d t
\end{aligned}
$$

and if $t_{u} \geq \frac{1}{2}$

$$
\begin{aligned}
\|N u\| & =N u\left(t_{u}\right)=\int_{0}^{t_{u}} \psi\left(\int_{t}^{t_{u}} a(s) \phi(u(s)) d s\right) d t \\
& \geq \int_{0}^{\frac{1}{2}} \psi\left(\int_{t}^{\frac{1}{2}} a(s) \phi(u(s)) d s\right) d t \\
& \geq\|u\| \psi^{-}(\|a\|) \int_{0}^{\frac{1}{2}}\left(\int_{t}^{\frac{1}{2}} s^{\beta} \frac{a(s)}{\|a\|} d s\right)^{\frac{1}{\alpha}} d t .
\end{aligned}
$$

Thus setting

$$
\begin{aligned}
D_{\phi}^{-}= & \min \left(\psi^{-}(\|a\|) \int_{0}^{\frac{1}{2}}\left(\int_{t}^{\frac{1}{2}} s^{\beta} \frac{a(s)}{\|a\|} d s\right)^{\frac{1}{\alpha}} d t\right. \\
& \left.\psi^{-}(\|a\|) \int_{\frac{1}{2}}^{1}\left(\int_{\frac{1}{2}}^{t}(1-s)^{\beta} \frac{a(s)}{\|a\|} d s\right)^{\frac{1}{\alpha}} d t\right),
\end{aligned}
$$

we get from the above that for all $u \in P,\|N u\| \geq D_{\phi}^{-}\|u\|$.

Remark 4.4. See that

$$
0<D_{\phi}^{-} \leq N_{P}^{-} \leq \lambda_{P}^{N} \leq \theta_{P}^{N} \leq N_{C}^{+} \leq D_{\phi}^{+}
$$


Lemma 4.5. Let

$$
O=\left\{u \in C_{X}: u^{\prime}(0)>0, u^{\prime}(1)<0 \text { and } u(x)>0 \forall x \in(0,1)\right\} .
$$

Then $O$ is open in $X$ and $N_{X}\left(C_{X}^{*}\right) \subset O$.

Proof. We have $O^{c}=F_{1} \cup F_{2} \cup F_{3}$ where

$$
\begin{aligned}
& F_{1}=\{u \in X: \text { there exists } x \in] 0,1[u(x) \leq 0\}, \\
& F_{2}=\left\{u \in X: u^{\prime}(0) \leq 0\right\} \text { and } \\
& F_{3}=\left\{u \in X: u^{\prime}(1) \geq 0\right\} .
\end{aligned}
$$

It is clear that $F_{2}$ and $F_{3}$ are closed sets in $X$ so let $\left(u_{n}\right) \subset F_{1}$ tending to $u$ in $X$ and $\left.\left(x_{n}\right) \subset\right] 0,1\left[\right.$ tending to $\bar{x} \in[0,1]$ with $u_{n}\left(x_{n}\right) \leq 0$. We distinguish the following cases

- $\bar{x} \in] 0,1\left[\right.$ in a such situation $u(\bar{x})=\lim u_{n}\left(x_{n}\right) \leq 0$ and $u \in F_{1}$,

- $\bar{x}=0$ in this case we obtain

$$
u^{\prime}(0)=\lim \frac{u_{n}\left(x_{n}\right)}{x_{n}} \leq 0 \quad \text { and } \quad u \in F_{2} .
$$

- $\bar{x}=1$ in this case we obtain

$$
u^{\prime}(1)=\lim \frac{u_{n}\left(x_{n}\right)}{x_{n}-1} \geq 0 \quad \text { and } \quad u \in F_{3} .
$$
$h \in C_{X}^{*}$

Now, let us show $N_{X}\left(C_{X}^{*}\right) \subset O$. We deduce from Lemma 4.3 that for all

$$
\|N h\|>0 \text { and } N h(x) \geq \rho(x)\|N h\|>0 \forall x \in(0,1)
$$

and since $N h(1)=N h(0)=0$,

$$
(N h)^{\prime}(0) \geq 0 \text { and }(N h)^{\prime}(1) \leq 0 \text {. }
$$

If $(N h)^{\prime}(0)=0$ then after two integration we get

$$
N h(x)=-\int_{0}^{x} \psi\left(\int_{0}^{t} \phi(h(s)) d s\right) d t \leq 0
$$

which is impossible. $(N h)^{\prime}(1)=0$ leads to the same contradiction, So we have $(N h)^{\prime}(0) \geq 0,(N h)^{\prime}(1) \leq 0$ and $N h \in O$.

Taking in account Remark 4.4 we deduce immediately from Lemma 3.5 the following nonexistence result.

Corollary 4.6. Assume that (12) and one of the following conditions

$$
f(t, u) \geq \alpha u \quad \text { for all } t \in[0,1] \text { and } u>0 \text { with } \alpha D_{\phi}^{-}>1
$$

or

$$
f(t, u) \leq \alpha u \text { for all } t \in[0,1] \text { and } u>0 \text { with } \alpha D_{\phi}^{+}<1
$$

holds true then Problem (15) admits no positive solution. 
Corollary 4.7. Assume that (12) and the following condition

$$
f^{\infty} D_{\phi}^{+}<1<f_{0} D_{\phi}^{-}
$$

hold true then Problem (15) admits a positive solution.

Proof. Let $\epsilon>0$ be such that $\left(f_{0}-\epsilon\right) D_{\phi}^{-}>1>D_{\phi}^{+}\left(f^{\infty}+\epsilon\right)$. It follows from definitions of $f_{0}$ and $f^{\infty}$ that there exist $\delta>0$ and $C>0$ such that

$$
\begin{aligned}
& F u \geq\left(f_{0}-\epsilon\right) u \text { for all } u \in P \cap \partial B(0, \delta) \\
& F u \leq\left(f^{\infty}+\epsilon\right) u+G(u) \text { for all } u \in P
\end{aligned}
$$

where for all $u \in C, G(u)=C$. Since $0<D_{\phi}^{-} \leq \lambda_{P}^{N} \leq \theta_{P}^{N} \leq D_{\phi}^{+}$we deduce from Theorem 3.11 that Problem (15) admits a positive solution.

Remark 4.8. See that Corollary 4.7 does not cover the case where $f^{0}=0, f_{\infty}=$ $\infty$. Theorem 1.2 in [10] cover this situation when $a \equiv 1, f(t, 0)=0$ and $f(t, s)>0$ for all $s>0$ and $t \in[0,1]$.

Note also that Theorems 1.1 and 1.2 in [10] are established for more general boundary conditions.

Corollary 4.9. Assume that (12) and the following condition

$$
\psi^{+}\left(l^{\infty}\right) D_{\phi}^{+}<1<\psi^{-}\left(l_{0}\right) D_{\phi}^{-}
$$

hold true then Problem (15) admits a positive solution.

Proof. It is easy to see that (14) implies

$$
\psi^{-}(s) \leq \frac{\psi(s \phi(u))}{u} \leq \psi^{+}(s) \text { for all } s, u \in(0,+\infty) .
$$

Thus it follows from définition of $\phi_{0}$ for arbitrary $\epsilon>0$ there exists $\delta>0$ such that for all $t \in[0,1]$ and $u \in[0, \delta], f(t, u) \geq\left(l_{0}-\epsilon\right) \phi(u)$ and then

$$
\frac{\psi(f(t, u))}{u} \geq \frac{\psi\left(\left(l_{0}-\epsilon\right) \phi(u)\right)}{u} .
$$

Passing to the limit we get

$$
f_{0}=\liminf _{u \rightarrow 0}\left(\min _{t \in[0,1]} \frac{\psi(f(t, u))}{u}\right) \geq \liminf _{u \rightarrow 0} \frac{\psi\left(\left(l_{0}-\epsilon\right) \phi(u)\right)}{u} \geq \psi^{-}\left(l_{0}-\epsilon\right)
$$

then $f_{0} \geq \psi^{-}\left(l_{0}\right)$. In similar way we can prove that $f^{0} \leq \psi^{+}\left(l^{0}\right)$ and $\psi^{-}\left(l_{\infty}\right) \leq f_{\infty} \leq f^{\infty} \leq \psi^{+}\left(l^{\infty}\right)$ and Corollary 4.9 follows from Corollary 4.7 .

Theorem 4.10. Suppose that $\phi$ is homogeneous then $N$ admits a unique positive eigenvalue $\lambda_{N}$. Moreover if one of the following conditions 20 and 21 is satisfied

$$
\begin{array}{ll}
f(t, u)>\mu_{N} u \forall t \in[0,1] & \forall u>0 \\
f(t, u)<\mu_{N} u \forall t \in[0,1] & \forall u>0
\end{array}
$$

where $\mu_{N}=\left(\lambda_{N}\right)^{-1}$, then Problem (15) admits no positive solutions and if one of the following situations

$$
f^{0}<\mu_{N}<f_{\infty} \leq f^{\infty}<\infty
$$




$$
f^{\infty}<\mu_{N}<f_{0} \leq f^{0}<\infty
$$

holds then Problem (15) admits a positive solution.

Proof. First note that the fact that $\phi$ is homogeneous implies that $N$ is positively 1-homogeneous, then taking in consideration Lemma 4.5, we deduce from Theorem 3.15 that $N$ admits a unique positive eigenvalue $\lambda_{N}$ and $\mu_{N}=$ $\left(\lambda_{C}^{N}\right)^{-1}=\left(\theta_{C}^{N}\right)^{-1}$. Nonexistence follows directly from Lemma 3.5.

Moreover $f^{0}<\mu_{N}<f_{\infty} \leq f^{\infty}<\infty$ (the other case is checked similarly) implies that there exists $\varepsilon>0$ small enough and positive constants $C_{1}, C_{2}$ such that

$$
\begin{gathered}
F(u) \leq\left(\mu_{N}-\varepsilon\right) u+G(u) \quad \text { for all } \in K^{*} \cap B(0, \delta) \\
\left(\mu_{N}+\varepsilon\right) u-C_{1} \leq F(u) \leq\left(f^{\infty}+\varepsilon\right) u+C_{2} \quad \text { for all } u \in K^{*}
\end{gathered}
$$

where $G u(t)=\max \left\{f\left(t, u(t)-f^{0} u(t), 0\right\}\right.$. Thus existence follows from Theorem 3.10

Remark 4.11. First note that the condition $\phi$ is homogeneous implies that there is $p>1$ such that for all $x \in \mathbb{R}, \phi(x)=\phi(1)|x|^{p-2} x$. The case $p=1$ and $a=1$ in Theorem 4.10 has been obtained in [2] (see Proposition 3.2 and Corollary 3.7). We can say that Theorem 4.10 generalize this principle of existence and nonexistence of positive solution following the position of the nonlinearity $f$ about the linearity $\mu_{p} u$ where $\mu_{p}$ is the first eigenvalue of the $p-$ Laplacian.

Remark 4.12. Note that in the case $a \equiv 1$, Theorem 4.10, does not cover the situations $f^{0}=0, f_{\infty}=\infty$ and $f_{0}=\infty, f^{\infty}=0$. Theorems 1.1 and 1.2 in [10] answer to these situations when $a \equiv 1, f(t, 0)=0$ and $f(t, s)>0$ for all $s>0$ and $t \in[0,1]$.

\section{2. $\phi$-Laplacian BVP with mixed boundary conditions}

In the above subsection we have find many difficulties to apply Theorems 3.12 and 3.13. In this section we present and application of these two theorems to a $\phi$-Laplacian BVP with mixed boundary conditions.

Consider the $\phi$-Laplacian BVP

$$
\left\{\begin{array}{l}
-\left(\phi\left(u^{\prime}\right)\right)(t)=a(t) f(t, u(t)) \quad t \in(0,1) \\
u(0)=u^{\prime}(1)=0
\end{array}\right.
$$

and let for $p>1, \mu_{p}$ be the first eigenvalue of

$$
\left\{\begin{array}{l}
-\left(\phi_{p}\left(u^{\prime}\right)\right)^{\prime}(t)=\mu a(t) \phi_{p}(u(t)) \quad t \in(0,1) \\
u(0)=u^{\prime}(1)=0 .
\end{array}\right.
$$

where $\phi_{p}(x)=|x|^{p-2} x$.

Let

$$
Y=\left\{u \in C^{1}([0,1]), u(0)=u^{\prime}(1)=0 .\right\}
$$

equipped with $C^{1}$-norm denoted $\|\cdot\|_{1}\left(\right.$ for $\left.u \in Y,\|u\|_{1}=\max \left(\|u\|,\left\|u^{\prime}\right\|\right)\right)$. From Ascoli Arzela Theorem the embedding $i_{Y}: Y \rightarrow E$ is compact. It is easy 
to see that

$$
P=\{u \in C, u(x) \geq x\|u\| \quad \forall x \in[0,1]\}
$$

is a cone in $E$ where for $x \in[0,1]$, and $C_{Y}=C \cap Y=i_{Y}^{-1}(C)$ is a cone in $Y$.

Let $A, N: E \rightarrow E$ be the operators defined for $h \in E$ by

$$
\begin{aligned}
& N h(x)=\int_{0}^{x} \psi\left(\int_{t}^{1} a(s) \phi(h(s)) d s\right) d t \quad \text { for all } x \in[0,1] \\
& A u(x)=\int_{0}^{x} \psi_{p}\left(\int_{t}^{1} a(s) \phi_{p}(u(s)) d s\right) d t \quad \text { for all } x \in[0,1]
\end{aligned}
$$

and $A_{Y}, N_{Y}: Y \rightarrow Y$ be the restrictions of $A$ and $N$ to $Y$. Arguing as in the above section we can see that each of $N$ and $N_{Y}$ are completely continuous operator and $u$ is a positive solution to (24) if and only if $u$ is a fixed point of the completely continuous operator NF. Moreover applying Theorem 3.15, by proving that $A\left(C^{*}\right) \subset \widetilde{O} \subset C_{Y}$ where

$$
\widetilde{O}=\left\{u \in Y, u(x)>0 \forall x \in(0,1] \text { and } u^{\prime}(0)>0\right\}
$$

is an open set in $Y$, we get that $A$ admits a unique positive eigenvalue $\lambda_{p}=$ $\lambda_{C}^{A}=\theta_{C}^{A}$ and $\left(\lambda_{p}\right)^{-1}=\mu_{p}$. As in the above section we have

- $N$ is increasing on $C$,

- $N$ is upper bounded on $C$ and $\|N u\| \leq M_{\phi}^{+}\|u\|$ for all $u \in C$ where

$$
M_{\phi}^{+}=\psi^{+}(\|a\|) \int_{0}^{1}\left(\int_{t}^{1} \frac{a(s)}{\|a\|} d s\right)^{\frac{1}{\beta}} d t,
$$

- $N$ is lower bounded on $P$ and $\|N u\| \geq M_{\phi}^{-}\|u\|$ for all $u \in P$ where

$$
M_{\phi}^{-}=\psi^{-}(\|a\|) \int_{0}^{1}\left(\int_{t}^{1} \frac{a(s) s^{\beta}}{\|a\|} d s\right)^{\frac{1}{\alpha}} d t .
$$

- $0<M_{\phi}^{-} \leq N_{P}^{-} \leq \lambda_{P}^{N} \leq \theta_{P}^{N} \leq N_{C}^{+} \leq M_{\phi}^{+}$.

It is clear that Corollaries 4.6, 4.7, 4.9 and Theorem 4.10 hold, we have also the following result which is derived from Theorems 3.12 and 3.13.

Theorem 4.13. Suppose that $\phi$ satisfies (12) and there exists $p>1$ such that

$$
\lim _{x \rightarrow+\infty} \frac{\phi(x)}{\phi_{p}(x)}=1,
$$

then if one of the following situations

$$
\begin{gathered}
f^{\infty} \max \left(M_{\phi}^{+}, \mu_{p}\right)<1<f_{0} M_{\phi}^{-} \\
f^{0} M_{\phi}^{+}<1<f_{\infty} \min \left(M_{\phi}^{-}, \mu_{p}\right) \text { and } f^{\infty}<\infty
\end{gathered}
$$

holds true, Problem (24) admits a positive solution. 
Proof. It follows from conditions (26) that

$$
\lim _{x \rightarrow+\infty} \frac{\psi(x)}{\psi_{p}(x)}=1
$$

where $\psi_{p}=\phi_{p}^{-1}$, then

$$
N u=A(u)+\circ(\|u\|) \quad \text { at } \infty .
$$

Moreover $f^{0} M_{\phi}^{+}<1<f_{\infty} \min \left(M_{\phi}^{-}, \mu_{p}\right)$ and $f^{\infty}<\infty$ (the other case is checked similarly) imply that there exists $\varepsilon>0$ small enough and positive constants $C_{1}, C_{2}$ such that

$$
\begin{gathered}
F(u) \leq\left(f^{0}+\varepsilon\right) u \quad \text { for all } \in C \cap B(0, \delta) \\
\left(f_{\infty}-\varepsilon\right) u-C_{1} \leq F(u) \leq\left(f^{\infty}+\varepsilon\right) u+C_{2} \quad \text { for all } u \in K^{*}
\end{gathered}
$$

and

$$
\left(f^{0}-\varepsilon\right) M_{\phi}^{+}<1<\left(f_{\infty}-\varepsilon\right) \min \left(M_{\phi}^{-}, \mu_{p}\right) .
$$

Applying Theorems 3.12 and 3.13, we deduce existence of a positive solution to Problem (24).

Remark 4.14. Let us return to the Dirichlet BVPs, and consider

$$
\left\{\begin{array}{l}
-\left(\phi\left(u^{\prime}\right)\right)(t)=g(u(t)) \quad t \in(0,2) \\
u(0)=u(2)=0
\end{array}\right.
$$

where $g \in C([0,+\infty(,[0,+\infty()$.

It is well known that if $u$ is a positive solution to (29) then $u$ is concave, symmetric about 1 and reaches its maximum at 1 . Hence, we deduce that $u$ is a positive solution to (29) if and only if the restriction of $u$ to $[0,1]$ satisfies

$$
\left\{\begin{array}{l}
-\left(\phi\left(u^{\prime}\right)\right)(t)=g(u(t)) \quad t \in(0,1) \\
u(0)=u^{\prime}(1)=0 .
\end{array}\right.
$$

So we can derive from the above existence and nonexistence results for positive solutions to Problem (29).

\section{References}

[1] Benmezai, A., Greaf, J.R., Kong, L.: Positive solution for the abstract Hammerstein equation and applications (submited)

[2] Benmezai, A.: Positive solutions for a second order two point boundary value problem. Commun. Appl. Anal. 14(2), 177-190 (2010)

[3] Benmezai, A., Djebali, S., Moussaoui, T.: Positive solution for $\phi$-Laplacian Dirichlet bvps. Fixed Point Theory 8(2), 167-186 (2007)

[4] Benmezai, A., Djebali, S., Moussaoui, T.: Multiple positive solution for $\phi$-Laplacian Dirichlet bvps. PanAm. Math. J. 17(3), 53-73 (2007)

[5] Chang, K.C.: A Nonlinear Krein Rutman theorem. J. Syst. Sci. Complex. 22, 542-554 (2009) 
[6] García-Huidobro, M., Manásevich, R., Zanolin, F.: A Fredholm-like result for strongly nonlinear second order O.D.E.'s. J. Differ. Equ. 114, 132-167 (1994)

[7] García-Huidobro, M., Manásevich, R., Zanolin, F.: On a pseudo Fučík spectrum for strongly nonlinear second order O.D.E.'s and an existence result. J. Comput. Appl. Math. 52, 219-239 (1994)

[8] García-Huidobro, M., Manásevich, R., Zanolin, F.: Strongly nonlinear second order O.D.E.'s with rapidly growing terms. J. Math. Anal. Appl. 202, $1-26$ (1996)

[9] García-Huidobro, M., Ubilla, P.: Multiplicity of solutions for a class of nonlinear second-order equations. Nonlinear Anal. TMA 28(9), 1509-1520 (1997)

[10] García-Huidobro, M., Manásevich, R., Ward, J.R.: Positive solutions for equations and systems with p-Laplace-like operators. Adv. Differ. Equ. 14(5-6), 401-432 (2009)

[11] Guo, D., Lakshmikantaham, V.: Nonlinear Problems in Abstract Cones. Academic Press, San Diego (1988)

[12] Mahadevan, R.: A note on a nonlinear Krein Rutman theorem. Nonlinear Analy. TMA 67, 3084-3090 (2007)

[13] Zeidler, E.: Nonlinear Functional Analysis and Its Applications, vol. I, Fixed Point Theorems. Springer, New York (1986)

Abdelhamid Benmezaï

Dynamical Systems Laboratory

Faculty of Mathematics

USTHB

PO Box 32

El-Alia Bab-ezouar

Algiers

Algeria

e-mail: abenmezai@yahoo.fr;

abenmezai@usthb.dz

Salima Mechrouk

Faculty of Sciences

University M'Hmed Bouguerra

Boumerdes

Algeria

e-mail: mechrouksalima@yahoo.fr

Received: 24 May 2011.

Accepted: 10 March 2012. 